\title{
Caracterización físico-química y clasificación del suelo de ladera manejado bajo el sistema Milpa Intercalada con Árboles Frutales
}

\section{Physical-chemical characterization and classification of the hillside soil managed under the Milpa Intercropped with Fruit Trees system}

Juan Pablo Torres Zambrano ${ }^{1}$, José Isabel Cortés Flores², Antonio Turrent Fernández ${ }^{3}$, Victor Hugo Volke Haller ${ }^{2}$, Carlos Alberto Ortiz Solorio y Alfredo Jiménez López 5 .

${ }^{1}$ Docente investigador, Academia de Ingeniería en Innovación Agrícola Sustentable, Instituto Tecnológico Superior de Ciudad Serdán, Av. Instituto, Tecnológico S/N, Col la Gloria, 75520 Cd Serdán, Puebla, México. jtorres@cdserdan.tecnm.mx

2 Profesor investigador, Edafología, Fertilidad de suelos, Colegio de Postgraduados, Carretera México-Texcoco Km. 36.5, Montecillo, Texcoco 56230, Estado de México. México. jicortes@colpos.mx, vvolke@colpos.mx, ortiz@colpos.mx

3 Investigador emérito, Productividad de agrosistemas del Campo Experimental Valle de México, Instituto Nacional de Investigaciones Forestales, Agrícolas y Pecuarias; Carretera Texcoco-Los Reyes Km.13.5, Texcoco, Coatlinchan, 56250 Texcoco, Estado de México, México. turrent.antonio@inifap.gob.mx

${ }^{4}$ Profesor investigador, Edafología, Génesis de suelo, Colegio de Postgraduados, Carretera México-Texcoco Km. 36.5, Montecillo, Texcoco 56230, Estado de México. México. ortiz@colpos.mx

5 Profesor investigador, Fruticultura, Colegio de Postgraduados, Carretera México-Texcoco Km. 36.5, Montecillo, Texcoco 56230, Estado de México. México. lopezja@colpos.mx

Recibido: 15/feb/2021 Revisado: 30/abr/2021

Aceptado: 30/may/2021 Publicado: 30/jul/2021

Resumen En un agrosistema de la sierra Norte de Oaxaca, México, el suelo se caracteriza por su baja fertilidad, principalmente contenidos bajos en fósforo y bases intercambiables, de reacción fuertemente ácida, alta acidez intercambiable y está desprotegido contra la erosión hídrica. En el año 2007, se estableció un experimento con el sistema milpa intercalada con árboles frutales (MIAF) en una ladera con una pendiente de $29.8 \%$. Después de seis años, la topografía cambió por efecto del laboreo y del manejo agronómico de las especies. El objetivo del trabajo fue la caracterización física y química del suelo del módulo MIAF después de seis años de estudio, así como definir su clasificación de acuerdo a la Taxonomía de Suelos y de la Base Mundial del Recurso Suelo. El análisis comprendió las zonas receptora y donante del módulo MIAF. Se realizaron perfiles de suelo en tres posiciones sobre el ancho de la ladera que comprendió el sitio experimental. Los resultados indicaron que la zona receptora de sedimentos y el área donde descansa el filtro de escurrimientos del muro vivo, mejoraron las propiedades físicas y químicas del suelo con respecto a la zona donante. El suelo se clasificó como Typic hapludults y Acrisol Háplico (Crómico).

Palabras clave zona donante y receptora; módulo MIAF; suelo ácido; Ultisol y Acrisol.

Abstract In an agrosystem in the northern highlands of Oaxaca, Mexico, the soil is characterized by its low fertility, mainly low phosphorus contents and exchangeable bases, strongly acid reaction, high exchangeable acidity and is unprotected against water erosion. In 2007, an experiment was established with the milpa system intercropped with fruit trees (MIAF) on hillside with a slope of $29.8 \%$. After six years, the topography changed due to tillage and agronomic management of the species. The objective of the work was the physical and chemical characterization of the soil of the MIAF module after six years of study, as well as defining its classification according to the Soil Taxonomy and the World Base of Soil Resources. The analysis comprised the recipient and donor areas of the MIAF module. Soil profiles were made in three positions over the width of the slope that comprised the experimental site. The results indicated that the sediment receiving area and the area where the runoff filter of the living wall rests, improved the physical and chemical properties of the soil with respect to the donor area. The soil was classified as Typic hapludults and Acrisols Haplic (Chromic).

Keywords recipient and donor areas; MIAF module; acid soil; Ultisols and Acrisols

Teknos Revista Científica. | Volumen 21 No.2 - diciembre 2021 | ISSN 1900-7388 (papel) | ISSN 2539-2190 (digital)

DOI: https://doi.org/10.25044/issn.2539-2190 


\section{Introducción}

Las prácticas de producción de la milpa de ladera en las regiones del estado de Oaxaca como la rozatumba-quema (RTQ) y roturación del suelo (aradura) en pequeñas unidades de producción, dan lugar a la pérdida de la fertilidad del suelo, rendimientos bajos, erosión hídrica del suelo y migración de la gente a otros estados y países (Cortés, et al., 2005). Además, el uso inadecuado del suelo en ladera provoca cambios negativos en sus propiedades, que llegan a afectar su capacidad productiva (Medina et al., 2008).

La fertilidad del suelo en laderas con pendientes mayores al 20 por ciento, en las regiones Mazateca, Cuicateca y Mixe de la Sierra Norte de Oaxaca, es agravada por el escaso uso de abonos orgánicos y químicos. Las bajas concentraciones de fósforo disponible, el bajo $\mathrm{pH}$ y elevada acidez intercambiable y la baja saturación de bases, son comunes en los suelos en las tres regiones, principalmente en Mazateca y Mixe. En contraste, los contenidos medios de materia orgánica de 3 a $6 \%$, permiten deducir que la condición física de estos suelos estaría menos amenazada a la degradación (Vergara et al., 2005). Sin embargo, en suelos donde el horizonte $A$ ha desaparecido por efecto de la erosión hídrica, es más apremiante la necesidad de proteger y conservar el suelo que aún persiste en estas regiones con prácticas que aborden la problemática de manera integral.

Los sistemas agroforestales tienen la capacidad de mejorar el suelo, mantener la productividad mediante un manejo planificado, racionalizando el impacto sobre el ambiente (Shibu, 2009). También, se les considera que pueden imitar características de los ecosistemas naturales, en particular aquellas que tienen efectos beneficiosos sobre las propiedades del suelo (Tornquist et al., 1999). El uso de sistemas agroforestales y la preparación adecuada del suelo en laderas, permiten el uso de cantidades bajas de fertilizantes que en combinación con orgánicos, pueden corregir la degradación química del suelo resultante del cultivo continuo; al mismo tiempo, también pueden aumentar la eficiencia del uso de fertilizantes (Smith et al., 2012).

Las tecnologías como el cultivo en callejones (Steppler y Nair, 1987) y la del pasto vetiver (Vetiveria zizanioides) (Dalton et al., 1996), derivadas de sistemas agroforestales, han demostrado eficiencia en el control de la erosión hídrica del suelo en laderas roturadas (Andrade y Rodríguez, 2002), y sus principios fueron considerados para la generación de una tecnología para el desarrollo gradual de terrazas en el trópico subhúmedo de México. La terraza de muro vivo (TMV), la cual consiste en establecer hileras a curva a nivel de una leguminosa como Gliricidia cepium y entre las hileras la siembra de maíz, con los residuos de cosecha y material podado depositados al pie y aguas arriba de las hileras de Gliricidia es una de ellas. La formación gradual de las terrazas en un periodo de seis años, en una ladera con una pendiente inicial de $14.5 \%$ disminuyó a $6.5 \%$. El proceso de formación fue a través de efectos combinados como la fuerza erosiva de la lluvia, la roturación unidireccional, el seto como muro vivo y el filtro de sedimentos compuesto de los residuos de cosecha y material podado de los árboles de Gliricidia (Turrent et al., 1995 y 1998).

El sistema milpa intercalado con árboles frutales (MIAF) en laderas, es el resultado de la combinación de las tecnologías TMV y MIAF en zona planas (Turrent et al., 1998; Cortés et al., 2004). Por tanto, es un sistema agroforestal de cultivo intercalado, constituido por tres especies, el árbol frutal, el maíz y el frijol $u$ otra especie comestible de porte bajo, en intensa interacción agronómica y que tiene como propósitos, la producción de maíz y frijol que son estratégicos para la seguridad alimentaria de las familias rurales, incrementar de manera significativa el ingreso neto familiar, controlar la erosión hídrica del suelo y con ello lograr un uso más eficiente del agua de lluvia al mejorar la calidad física, química y biológica del suelo (Cortés et al., 2012; Cortés et al., 2005).

En el año 2007, se inició la segunda fase del "Proyecto Manejo Sustentable de Laderas" (PMSL) en

Teknos Revista Científica. | Volumen 21 No.2 - diciembre 2021 | ISSN 1900-7388 (papel) | ISSN 2539-2190 (digital)

DOI: https://doi.org/10.25044/issn.2539-2190 
un agrosistema representativo en la comunidad de Santa María Tlahuitoltepec, Mixe, Oax. (Ramos et al., 2007). El objetivo del proyecto fue el mejoramiento de la tecnología MIAF y la obtención de las recomendaciones de fertilizaciones específicas para este agrosistema. A los seis años de estudio, el sistema MIAF generó modificaciones positivas en el suelo, disminuyó la pendiente, dando lugar a la formación paulatina de terrazas con las labores anuales de campos normales (Salinas, 2015), controló la erosión hídrica del suelo e incrementó la infiltración de agua. Sin embargo, Camas (2011) recomienda que el manejo de la ladera debe ser por secciones (parta alta, media y baja) debido a la variación de fertilidad del suelo y además dentro del módulo MIAF, debe haber un manejo específico por el reacomodo de sedimentos en las diferente zonas del módulo.

Por tanto, el objetivo del presente trabajo fue la caracterización física y química del suelo del módulo MIAF después de seis años de estudio y así mismo, definir la clasificación del suelo con base a la Taxonomía de Suelos y de la Base Mundial del Recurso Suelo.

\section{Metodología}

\subsection{Sitio experimental}

El trabajo experimental se llevó a cabo en un agrosistema representativo de la región Mixe Alta en la Sierra Norte de Oaxaca, en el periodo 2007 al 2012 en la ranchería Santa Cruz, del municipio de Santa María Tlahuitoltepec. El sitio se ubica a $17^{\circ} 05^{\prime} 18.45^{\prime \prime} \mathrm{N}$, $96^{\circ} 02^{\prime} 53.56 " \mathrm{O}$ y a una altitud de $2,300 \mathrm{~m}$. El clima según García (2004) es templado subhúmedo (Cw) con lluvias abundantes en verano y con una precipitación promedio de $1375 \mathrm{~mm}$. La temperatura media anual es de $15.2^{\circ} \mathrm{C}$, siendo los meses más fríos noviembre, diciembre, enero y febrero (García, 2004; INEGI, 2010).

La geología dominante es de origen volcánico, principalmente de rocas ígneas, toba ácida (52.39\%) y andesita (30.50\%); sedimentarias, caliza (14.60\%) y metamórficas, esquisto $(2.51 \%)$. Los suelos se han formado principalmente en las interfaces de rellenos formados por tobas andesíticas con predominio de suelos residuales y superficiales, alterados fuertemente por el interperismo químico (INEGI, 2010). Con datos de las normales climatológicas de la estación Tlahuitoltepec (Ortiz et al., 2011) y el programa Newhall.1.5.1 ${ }^{\circledR}$ (USDA, 2011), se determinó que el suelo es de un régimen de humedad údico y un régimen de temperatura isomésico.

En el sitio se estableció un experimento con el sistema MIAF en una ladera con una pendiente inicial de $29.8 \%$ (Salinas, 2015) y el suelo está clasificado como Acrisol (INEGI, 2010). En el mes de enero del año 2007, se realizó un análisis del suelo de acuerdo a la metodología de Van Reeuwijk (1999). El suelo es de textura franco limoso en la profundidad de 0 a 0.20 $\mathrm{m}$ y franco arcillo-limoso de 0.20 a $0.40 \mathrm{~m}$; tiene un $\mathrm{pH}$ que va de moderadamente ácido en los primeros 0.20 $\mathrm{m}$ y fuertemente ácido hasta los $0.40 \mathrm{~m}$; el contenido de materia orgánica es bajo a la profundidad de 0.20 $\mathrm{m}$ y muy bajo a los $0.40 \mathrm{~m}$; la capacidad de intercambio catiónico $(\mathrm{ClC})$ varía de media a muy alta a los 0.20 y $0.40 \mathrm{~m}$. Los contenidos de potasio $(\mathrm{K})$ y fósforo $(\mathrm{P})$ fueron trazas, y en calcio $(\mathrm{Ca})$ y magnesio $(\mathrm{Mg})$ muy bajos en ambas profundidades.

Se establecieron dos sub-experimentos: 1) maíz como mesocultivo y 2) árbol de durazno como epicultivo, además de una leguminosa (sotocultivo) de exploración que acompaña al maíz en el arreglo topológico de surcos alternos con micro-rotación anual. Se trazaron dos módulos MIAF, con hileras a curvas a nivel separadas entre ellas a $10.6 \mathrm{~m}$. Cada módulo MIAF tiene ocho surcos de $0.80 \mathrm{~m}$ de ancho destinados para el maíz y la leguminosa, cuatro aguas arriba y cuatro aguas abajo con respecto a la hilera de los árboles frutales, con una anchura de $4.2 \mathrm{~m}$. En la parte central de la hilera fueron plantados los árboles de durazno variedad "CP 2005" a una densidad de 943 árboles ha-1 ${ }^{-1}$ con una separación de $1.0 \mathrm{~m}$ entre ellos sobre la hilera $(10.6 \times 1.0 \mathrm{~m})$ (Figura 1).

En el sub-experimento maíz se estudió la respuesta del rendimiento de grano a los factores $\mathrm{N}$, $\mathrm{P}, \mathrm{K}$ y densidad de población (D), cada uno a cinco niveles: $\mathrm{N}$ a 40, 80, 120160 y $200 \mathrm{~kg} \mathrm{ha}^{-1} ; \mathrm{P}_{2} \mathrm{O}_{5}$ a 30 , $60,90,120$ y $150 \mathrm{~kg} \mathrm{ha}^{-1} ; \mathrm{K}_{2} \mathrm{O}$ a 0, 20, 40, 60 y $80 \mathrm{~kg}$

Teknos Revista Científica. | Volumen 21 No.2 - diciembre 2021 | ISSN 1900-7388 (papel) | ISSN 2539-2190 (digital)

DOI: https://doi.org/10.25044/issn.2539-2190 
ha $^{-1}$ y D a $40,50,60,70$ y 80 mil plantas ha-1; con los cuales se obtuvieron 26 tratamientos a partir del diseño de hipercubo doble lotificado (Volke et al., 2005; Martínez y Martínez, 1996). Los 26 tratamientos están distribuidos en el terreno bajo del diseño experimental de parcelas divididas con dos repeticiones. La parcela grande es la posición de cada surco, con respecto a la hilera del árbol frutal. La parcela chica comprende los 26 tratamientos antes mencionados. La parcela experimental consistió de cuatro surcos de maíz, dos aguas arriba y dos aguas abajo respecto a la hilera de los frutales, cada uno de $4.0 \mathrm{~m}$ de longitud $\times 0.8 \mathrm{~m}$ de ancho y como parcela útil se consideró los $3.0 \mathrm{~m}$ centrales de cada surco.

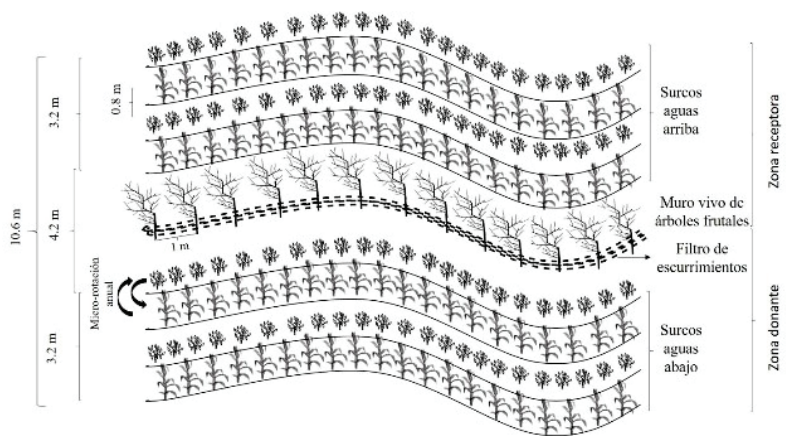

Figura 1. Modelo del módulo milpa intercalada con árboles frutales (MIAF) en una ladera; arreglo topológico en surcos alternos con micro-rotación anual de maíz y frijol; y ubicación de las zonas donante y receptora de sedimentos.

Para el sub-experimento árbol de durazno se estudiaron también cuatro factores con cinco niveles cada uno: $\mathrm{N}$ con 17, 24, 31, 38 y $45 \mathrm{~g}_{\text {árbol }}{ }^{-1} ; \mathrm{P}_{2} \mathrm{O}_{5}$ con $5,10,15,20$ y 25 g árbol- $^{-1} ; \mathrm{K}_{2} \mathrm{O}$ con $17,24,31,38 \mathrm{y}$ $45 \mathrm{~g}$ árbol $^{-1} \mathrm{y}$ estiércol con $0,0.5,1.0,1.5$ y $2.0 \mathrm{~kg}$ árbol $^{-1}$, por año de edad del árbol hasta el tercer año (de ahí en adelante se mantuvieron constantes), combinados también en 26 tratamientos, de acuerdo al diseño de tratamientos hipercubo doble. La parcela experimental fue de cuatro árboles y como parcela útil se consideraron los dos árboles centrales.

El muestreo del suelo para la caracterización física y química del módulo MIAF fue en el año 2013 a 5 semanas previas a la fecha de siembra (2 marzo). En el área de las especies anuales, cuya pendiente había disminuido a $20.4 \%$, por efecto del laboreo, los escurrimientos y la formación paulatina de la terrazas (Salinas, 2015), correspondiente al tratamiento central del diseño en estudio para el maíz (120-90-40 kg de $\mathrm{N}-\mathrm{P}_{2} \mathrm{O}_{5}-\mathrm{K}_{2} \mathrm{O}$ ha $^{-1}$ ), por ser el de referencia y centro del diseño. Además, año con año se aplicó $2 \mathrm{t} \mathrm{ha}^{-1} \mathrm{de}$ estiércol de pollo en el surco S1 de maíz bajo microrotación anual. Se obtuvieron tres sub-muestras de suelo de la parcela útil por surco a dos profundidades: 0-0.20 y $0.20-0.40 \mathrm{~m}$, en las dos repeticiones del experimento.

En la hilera de los árboles de durazno, aguas arriba se estuvo colocando el filtro de escurrimientos compuesto de rastrojo de maíz, madera podada de los árboles y malezas segadas. El tratamiento de estudio del árbol de durazno, también correspondió al central del diseño (93-45-93 g de $\mathrm{N}-\mathrm{P}_{2} \mathrm{O}_{5}-\mathrm{K}_{2} \mathrm{O}$ y $3 \mathrm{~kg}$ de pollinaza árbol ${ }^{-1}$ ). Se obtuvieron muestras de suelo a dos profundidades: de 0 a 0.10 y 0.10 a $0.30 \mathrm{~m}$, en la parcela útil de las dos repeticiones. La primera capa tuvo un espesor de $0.10 \mathrm{~m}$, porque es el resultado de los depósitos de sedimentos y acumulación de materiales orgánicos ya en descomposición; así que la profundidad de 0.10 a $0.30 \mathrm{~m}$ viene siendo el suelo original del sitio.

También, se excavaron tres perfiles de suelo a lo ancho del sitio experimental (Figura 2). El perfil 1 se ubicó en la orilla de la parte Norte del terreno y entre las hileras de los árboles de durazno, en una zona donde la pendiente es cóncava y el suelo superficial con un color blanquecino; el segundo perfil hacia el centro del terreno donde la pendiente es convexa y el suelo es rojizo intenso, y el tercer perfil casi al final de las hileras (parte Sur) con la pendiente cóncava, con color rojizo no tan intenso. Los tres perfiles se describieron con la metodología propuesta por Cuanalo (1981). Además, se colectaron muestras alteradas de cada uno de los estratos identificados para sus análisis físicos y químicos.

Las muestras compuestas de suelo del módulo MIAF y alteradas de los perfiles, se secaron a la sombra, se molieron con martillo de madera y se cribaron en un tamiz de $2 \mathrm{~mm}$. En ellas se llevaron a cabo determinaciones físicas y químicas. En las muestras del módulo MIAF, los análisis se realizaron de acuerdo a los procedimientos del Laboratorio de 
Fertilidad de Suelos del Colegio de Postgraduados (Etchevers, 1992) para determinar pH, MO, N, P, K, $\mathrm{Ca}, \mathrm{Mg}, \mathrm{CIC}$ y acidez intercambiable; y para la determinación de CC, PMP, Da y textura fue de acuerdo a la Norma Oficial Mexicana (NOM-021RECNAT-2000) (SEMARNAT, 2000).

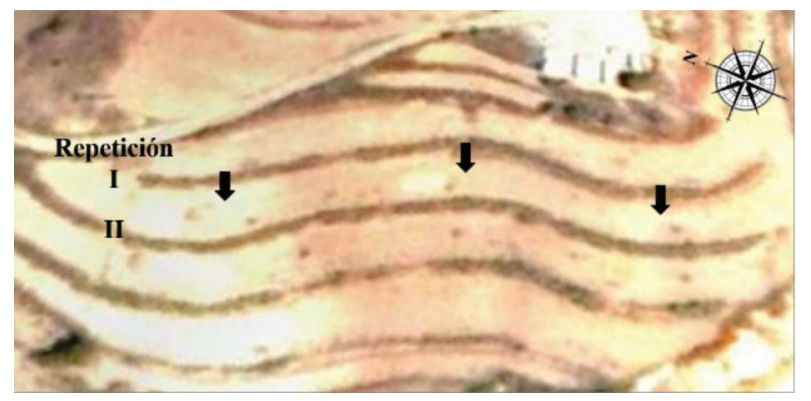

Figura 2. Ubicación de los perfiles del suelo en el sitio experimental Santa Cruz, Tlahuitoltepec, Oax., dentro del sistema milpa intercalada con árboles frutales (MIAF) en una ladera al sexto año de su establecimiento.

En las muestras de los perfiles, los análisis se realizaron de acuerdo a los procedimientos descritos por Van Reeuwij (1999) en el laboratorio de Génesis, Morfología y Clasificación de Suelos del Colegio de Postgraduados. Con la información de campo recabada de los perfiles y del laboratorio, se clasificaron los suelos a nivel subgrupo con las Claves para la Taxonomía del Suelo (SSS, 2014), y con la Base Referencial Mundial del Recurso suelo (WRB, 2007).

\section{Resultados y discusión}

\subsection{Características físicas del suelo}

En los cuatro surcos de la zona donante del módulo MIAF, el suelo a la profundidad de 0 a 0.20 m muestra un cambio en la textura con respecto a los cuatro surcos de la zona receptora, es decir de clase arcilloso (Tabla 1). Esto se puede atribuir a la roturación y laboreo del suelo, lo que propició el reacomodo del horizonte Ap de la zona donante a la zona receptora del siguiente módulo. El contenido de humedad en la zona receptora a la profundidad de 0 a $0.20 \mathrm{~m}$ presentó en promedio $17.2 \%$, mientras que en la zona donante fue de $27.31 \%$. A la profundidad de 0.20 a $0.40 \mathrm{~m}$, el contenido de humedad promedio también fue mayor en la zona donante que en la receptora, con 32.52 y $26.07 \%$, respectivamente (Tabla 1 ). Por lo tanto, debido al reacomodo del suelo en la zona receptora, ésta presenta mejores condiciones de textura que permite una mejor infiltración respecto a la zona donante a la profundidad de 0 a $0.20 \mathrm{~m}$.

En el área del filtro de escurrimientos de la hilera de los árboles frutales, que también corresponde al área receptora, el contenido de humedad a la profundidad de 0.10 a $0.30 \mathrm{~m}$, fue similar que en los surcos de la zona receptora a la profundidad de 0 a $0.20 \mathrm{~m}$, así como en el tipo de textura, dado que es el suelo sin perturbación y con la pendiente inicial.

La densidad aparente en la zona receptora a la profundidad de $0.20 \mathrm{~m}$ tuvo una disminución en el surco 4 respecto a los surcos 1, 2 y 3 (Tabla 1), probablemente se deba a que es el último surco próximo al área no perturbada de la franja de los árboles frutales. El paso de la yunta, está limitado por las ramas aguas arriba de los árboles de durazno, razón por la que no se puede roturar en esta zona. Lo anterior, provoca que se forme una línea de acumulación de suelo entre el límite del área de los árboles y el último surco aguas arriba, como lo describe Salinas (2015) en la lectura topográfica de la sección transversal del terreno en estudio. En la zona donante del módulo a la profundidad de 0 a $0.20 \mathrm{~m}$, la densidad aparente de los surcos 5 y 6 se incrementa respecto a los surcos 7 y 8 (Tabla 1 ), debido a que son los surcos con mayor desprendimiento de suelo por las labores de roturación y de cultivo.

En este agrosistema, al suelo se le considera con humedad residual, debido a que los productores siembran maíz y frijol en los primeros días de marzo y las lluvias inician en el periodo mayo-junio. En la siembra, las semillas se depositan a una profundidad entre 0.10 y $0.20 \mathrm{~m}$ para encontrar la humedad residual. En este contexto, el contenido de humedad al momento del muestreo, indicó que en la zona donante a la profundidad de 0.20 a $0.40 \mathrm{~m}$ en los surcos 6 y 7 , se encontraba cercana a la CC (33.73\%) con $31.34 \%$ en promedio; mientras en el surco 5 
estuvo $3.69 \%$ por arriba de su CC; y en el surco 8 fue $4.74 \%$ menor respecto a su CC (Tabla1).

Por otro lado, en la zona receptora del módulo, el suelo a la profundidad de 0.20 a $0.40 \mathrm{~m}$, el contenido de humedad en los surcos 1,2 y 3 tuvieron una diferencia de $6.96,5.65$ y $4.23 \%$, respectivamente, por debajo de su CC; y en el surco 4 a la misma profundidad, el contenido de humedad es similar a su CC (Tabla 1). Sin embargo, en el área del filtro de escurrimientos, el contenido de humedad a la profundidad de 0.10 a $0.30 \mathrm{~m}$, está por debajo de su PMP en un $4.20 \%$ (Tabla 1), esto se puede atribuir a la demanda de agua por parte de los árboles de durazno, ya que en estas fechas, los frutos iniciaban su crecimiento y son altamente consumidores de agua (Ryugo, 1993). Ante esto, la absorción de agua por las raíces de los árboles de durazno para satisfacer su demanda durante el crecimiento de frutos y el vegetativo, proviene de estratos más profundos (Lott et al., 2003), entre 0.40 y $1.0 \mathrm{~m}$, como lo reporta Torres (2006) y Santiago (2006), en el sistema MIAF con árboles de duraznos en suelos con pendiente somera $(5 \%)$ y pronunciada $(37 \%)$, respectivamente.

Tabla 1. Características físicas del suelo del módulo milpa intercalada con árboles frutales (MIAF) en una ladera en San Cruz, Tlahuitotepec, Oax.

\begin{tabular}{|c|c|c|c|c|c|c|c|c|c|c|}
\hline \multirow[t]{2}{*}{ Sección del modulo } & \multirow[t]{2}{*}{ Surco } & \multirow{2}{*}{$\begin{array}{c}\text { Profundidad } \\
(\mathrm{m})\end{array}$} & \multirow{2}{*}{$\begin{array}{c}\operatorname{Hum}^{2} \\
(\%)\end{array}$} & \multirow{2}{*}{$\begin{array}{c}\mathrm{Da}^{\mathrm{Y}} \\
\left(\mathrm{g} \mathrm{cm}^{-3}\right) \\
\end{array}$} & \multirow{2}{*}{\multicolumn{2}{|c|}{$\begin{array}{c}\mathrm{CC}^{\mathrm{X}} \quad \mathrm{PMPW}^{\mathrm{W}} \\
--\mathrm{-}^{-} \% \\
\end{array}$}} & \multirow{2}{*}{$\begin{array}{c}\text { Fracción } \\
\mathrm{A}^{\vee} \\
\end{array}$} & \multirow{2}{*}{$\frac{(\%)}{L^{U}}$} & \multirow{2}{*}{\multicolumn{2}{|c|}{ Clase textural }} \\
\hline & & & & & & & & & & \\
\hline \multirow{8}{*}{ Receptora } & \multirow[t]{2}{*}{1} & $0-0.20$ & 15.73 & 1.27 & 38.15 & 17.80 & 32.00 & 31.00 & 37.00 & Franco arcilloso \\
\hline & & $0.20-0.40$ & 23.34 & 1.34 & 30.30 & 20.50 & 29.00 & 21.00 & 50.00 & Arcilloso \\
\hline & \multirow[t]{2}{*}{2} & $0-0.20$ & 19.24 & 1.26 & 30.25 & 20.45 & 33.50 & 27.50 & 39.00 & Franco arcilloso \\
\hline & & $0.20-0.40$ & 26.70 & 1.35 & 32.35 & 22.00 & 28.50 & 23.50 & 48.00 & Arcilloso \\
\hline & \multirow[t]{2}{*}{3} & $0-0.20$ & 17.70 & 1.29 & 28.65 & 19.15 & 33.50 & 27.50 & 39.00 & Franco arcilloso \\
\hline & & $0.20-0.40$ & 25.62 & 1.39 & 29.85 & 20.20 & 33.50 & 21.50 & 45.00 & Arcilloso \\
\hline & \multirow[t]{2}{*}{4} & $0-0.20$ & 16.69 & 1.17 & 29.00 & 18.90 & 29.50 & 31.50 & 39.00 & Franco arcilloso \\
\hline & & $0.20-0.40$ & 28.56 & 1.29 & 28.35 & 19.50 & 32.50 & 28.50 & 39.00 & Franco arcilloso \\
\hline \multirow{5}{*}{ Filtro de escurrimientos } & & $0-0.10$ & 11.84 & 1.24 & 33.00 & 21.50 & 42.00 & 28.00 & 30.00 & Franco arcilloso \\
\hline & & $0.10-0.30$ & 16.64 & 1.31 & 28.70 & 20.60 & 34.00 & 26.00 & 40.00 & Franco arcilloso \\
\hline & \multirow[t]{2}{*}{5} & $0-0.20$ & 31.07 & 1.30 & 33.40 & 23.50 & 24.50 & 23.50 & 52.00 & Arcilloso \\
\hline & & $0.20-0.40$ & 38.14 & 1.29 & 34.45 & 26.75 & 23.50 & 24.50 & 52.00 & Arcilloso \\
\hline & \multirow[t]{2}{*}{6} & $0-0.20$ & 23.49 & 1.30 & 31.65 & 22.20 & 27.50 & 23.50 & 49.00 & Arcilloso \\
\hline \multirow{5}{*}{ Donante } & & $0.20-0.40$ & 31.63 & 1.31 & 33.80 & 24.60 & 24.50 & 22.50 & 53.00 & Arcilloso \\
\hline & \multirow[t]{2}{*}{7} & $0-0.20$ & 26.87 & 1.23 & 32.25 & 22.30 & 21.50 & 28.50 & 50.00 & Arcilloso \\
\hline & & $0.20-0.40$ & 31.04 & 1.31 & 33.65 & 24.00 & 20.50 & 24.50 & 55.00 & Arcilloso \\
\hline & \multirow[t]{2}{*}{8} & $0-0.20$ & 27.79 & 1.24 & 32.70 & 22.50 & 25.50 & 25.50 & 49.00 & Arcilloso \\
\hline & & $0.20-0.40$ & 29.26 & 1.19 & 34.00 & 24.70 & 27.50 & 20.50 & 52.00 & Arcilloso \\
\hline
\end{tabular}

${ }^{\mathrm{Z}}$ Contenido de humedad. ${ }^{\mathrm{Y}}$ Densidad aparente. ${ }^{\mathrm{X}}$ Capacidad de campo. ${ }^{\mathrm{W}}$ Punto de marchitez permanente. ${ }^{\vee}$ Arena. ${ }^{\mathrm{U}}$ Limo $\mathrm{y}^{\top}$ Arcilla.

\subsection{Características químicas del suelo}

Las características químicas del suelo del módulo MIAF en las zonas receptora y donante, indican un $\mathrm{pH}$ medianamente ácido a las dos profundidades; la capacidad de intercambio catiónico $(\mathrm{CIC})$ es baja en las dos zonas, con excepción del surco 6 a la profundidad de $0.20-0.40 \mathrm{~m}$ donde es alta. En la zona del filtro de escurrimientos, la $\mathrm{CIC}$ a la profundidad 0-0.10 m es muy alta, y está relacionada por la cantidad de materia orgánica acumulada producto del filtro de escurrimientos, lo cual concuerda con lo encontrado por Camas (2011) en una ladera manejada con el sistema MIAF en el estado de Chiapas.

El contenido de materia orgánica $(\mathrm{MO})$ en la zona receptora a la profundidad $0-0.20 \mathrm{~m}$ y además en el surco 4 hasta la profundidad de 0.40 m es medio; en la zona donante los surcos 5, 6 y 7 van de contenido bajo a la profundidad de $0.20 \mathrm{~m}$ y muy bajo de 0.20 $0.40 \mathrm{~m}$ en los surcos 6 y 7 , para el surco 8 se ve 
favorecido con un contenido medio a la profundidad de $0.20 \mathrm{~m}$ y bajo hasta los $0.40 \mathrm{~m}$. En la zona de la franja del filtro de escurrimientos, el contenido de $\mathrm{MO}$ es alto a los $0.10 \mathrm{~m}$ de profundidad y medio de $0.10-0.30 \mathrm{~m}$, lo que era de esperarse por la incorporación de residuos para el filtro de escurrimientos.

El contenido de $\mathrm{P}$ es medio en los surcos 1 y 2 , y bajo en los surcos 3 y 4 a los primeros $0.20 \mathrm{~m}$ de profundidad, y a la profundidad de $0.20-0.40 \mathrm{~m}$ en los cuatro surcos varía de bajo a trazas. En la zona donante, en los surcos $5,6,7$ y 8 el contenido de $P$ es bajo en los primeros $0.20 \mathrm{~m}$ de profundidad y trazas hasta los $0.40 \mathrm{~m}$; y en la zona del filtro de escurrimientos, es alto a los $0.10 \mathrm{~m}$ de profundidad y bajo de 0.10 a $0.30 \mathrm{~m}$, que corresponde al suelo superficial original. El movimiento del suelo con $\mathrm{P}$ se debió al laboreo y los escurrimientos, siendo la zona receptora hasta el muro vivo beneficiados con $\mathrm{P}$ proveniente de fertilizantes y de la pollinaza aplicados (Sanyal et al., 2015). Además, en el área del filtro de escurrimientos el contenido alto de $\mathrm{P}$ se debió también a la no roturación del suelo, la aplicación continua de fertilizante $\mathrm{P}_{2} \mathrm{O}_{5}$ y estiércol de pollo a los árboles frutales, lo cual fue reportado por Camas et al., (2012) en el sistema MIAF en Chiapas.

El contenido de $\mathrm{K}$ en toda la zona receptora es alta en las dos profundidades, mientras en la zona donante en los primeros $0.20 \mathrm{~m}$ en los surcos 5 y 7 es medio $y$ en el resto de los surcos en ambas profundidades es alto. En cuanto al $\mathrm{Ca}$ y $\mathrm{Mg}$, en la zona receptora el contenido es bajo y en la zona donante de bajo a muy bajo; sin embargo, en el área del filtro de escurrimientos a los $0.10 \mathrm{~m}$ de profundidad es medio (Tabla 2). La aplicación de pollinaza y $P$, además de contribuir a neutralizar en parte la acidez intercambiable, aportar fósforo y bases intercambiables, coadyuvaría a mejorar la fertilidad del suelo, como lo reportan, Pool et al. (2000) y Vergara et al., (2005).

La acidez intercambiable del suelo en general es menor en la zona receptora respecto a la donante, principalmente a la profundidad de 0 a $0.20 \mathrm{~m}$ y en los primeros $0.10 \mathrm{~m}$ de profundidad en la zona del filtro de escurrimientos (Tabla 2). Lo niveles altos de acidez intercambiable normalmente se relacionan con escasez de bases intercambiables y de fósforo (Fassbender, 1986). Por tanto, lo sucedió en la zona receptora del módulo, la acidez intercambiable disminuyó por la aplicación continua de fósforo y el estiércol, principalmente en el surco 1, donde tuvo efectos en la disminución de la acidez hasta la profundidad de los $0.40 \mathrm{~m}$, lo anterior permite un sostenibilidad de la fertilidad en un suelo Ultisol como lo reportaron Ojo et al. (2015).

\subsection{Clasificación del suelo}

\subsubsection{Características morfológicas del suelo}

En las partes norte, media y sur de la ladera, el suelo es profundo (> $1.0 \mathrm{~m}$ ), con estructuras subangulares hasta aproximadamente $0.70 \mathrm{~m}$ de profundidad, es friable y de permeabilidad moderada. En la parte norte, el suelo a la profundidad de $0.20 \mathrm{~m}$ tiene un color marrón amarillento oscuro y en el resto del perfil el color es marrón oscuro. En la parte media el color marrón amarillento oscuro llega hasta la profundidad de $0.70 \mathrm{~m}$ y marrón oscuro en el resto del perfil. En la parte sur, a los $0.20 \mathrm{~m}$ de profundidad el suelo es también de color marrón y de ahí en adelante cambia a amarillo pardo (Tabla 3 ).

Estas condiciones morfológicas indican condiciones físicas favorables para el desarrollo de la raíz de las plantas. La presencia de raíces en todo el perfil, constata esto con una mayor proliferación en los primeros $0.30 \mathrm{~m}$.

\subsection{Características físicas y químicas del suelo}

La textura del horizonte Ap en los tres perfiles fue franco arcilloso; en los horizontes $\mathrm{Bw}, \mathrm{Bt}_{\text {y }} \mathrm{Bt}_{2}$ varió, predominando la textura franco arcillosa y franco limosa, seguido de la arcillosa y arcillo limosa, y migajón arcillo limoso. La densidad aparente en el horizonte Ap fue en general de $1.3 \mathrm{~g} \mathrm{~cm}^{-3}$, de 1.4 en el horizonte $\mathrm{Bw}$ y de 1.5 en el $\mathrm{Bt}$, en los tres perfiles (Tabla 4). 
Torres Zambrano, J. P., Cortés Flores, J. I., Fernández, A. T., Volke Haller, V. H., Ortiz Solorio, C. A., \& Jiménez López, A. (2021). Caracterización físico-química y clasificación del suelo de ladera manejado bajo el sistema Milpa Intercalada con árboles frutales. Teknos Revista Científica, 21(2), 35-45.

Tabla 2. Características químicas del suelo en el módulo milpa intercalada con árboles frutales (MIAF) en una ladera en San

\begin{tabular}{|c|c|c|c|c|c|c|c|c|c|c|c|}
\hline \multirow{5}{*}{$\begin{array}{l}\text { Sección del } \\
\text { modulo }\end{array}$} & Surco & Profundidad & $\mathrm{pH}^{\mathrm{z}}$ & $\mathrm{MO}^{\mathrm{Y}}$ & $\mathrm{N}^{\mathrm{X}}$ & $\mathrm{P}^{\mathrm{W}}$ & $\mathrm{K}^{\mathrm{V}}$ & $\mathrm{Ca}^{\mathrm{U}}$ & $\mathrm{Mg}^{\top}$ & $\mathrm{ClC}^{\mathrm{S}}$ & Acidez $^{R}$ \\
\hline & & $(\mathrm{m})$ & & $(\%)$ & $(\%)$ & $\left(\mathrm{mg} \mathrm{kg}^{-1}\right)$ & & \multicolumn{4}{|c|}{----------- $\mathrm{Cmol}(+) \mathrm{kg}^{-1}$--------- } \\
\hline & 1 & $0-0.20$ & 5.4 & 2.6 & 0.13 & 29.5 & 1.6 & 3.9 & 0.7 & 12.1 & 0.8 \\
\hline & & $0.20-0.40$ & 5.8 & 0.6 & 0.06 & 0.4 & 1.0 & 4.5 & 0.9 & 11.7 & 0.8 \\
\hline & 2 & $0-0.20$ & 5.6 & 2.5 & 0.12 & 16.2 & 1.1 & 4.2 & 0.7 & 11.6 & 0.8 \\
\hline \multirow{5}{*}{ Receptora } & & $0.20-0.40$ & 5.7 & 0.9 & 0.06 & $\operatorname{Tr}^{Q}$ & 1.0 & 3.8 & 0.7 & 12.2 & 1.5 \\
\hline & 3 & $0-0.20$ & 5.5 & 2.1 & 0.12 & 11.7 & 1.1 & 4.4 & 0.6 & 12.0 & 0.8 \\
\hline & & $0.20-0.40$ & 5.5 & 0.6 & 0.05 & $\operatorname{Tr}^{\mathrm{Q}}$ & 0.9 & 3.6 & 0.6 & 9.6 & 3.2 \\
\hline & 4 & $0-0.20$ & 5.5 & 3.1 & 0.16 & 14.7 & 1.2 & 4.2 & 0.6 & 10.7 & 0.8 \\
\hline & & $0.20-0.40$ & 5.5 & 2.4 & 0.14 & 0.9 & 0.9 & 3.6 & 0.5 & 11.7 & 1.1 \\
\hline \multirow{5}{*}{ Receptora } & Área del filtro de & $0-0.10$ & 6.4 & 4.0 & 0.27 & 52.9 & 5.0 & 9.1 & 1.6 & 86.1 & 0.1 \\
\hline & escurrimientos & $0.10-0.30$ & 5.1 & 2.3 & 0.13 & 0.9 & 1.2 & 3.2 & 0.4 & 14.2 & 1.6 \\
\hline & 5 & $0-0.20$ & 5.4 & 1.0 & 0.08 & 1.0 & 0.5 & 1.5 & 0.3 & 14.1 & 2.2 \\
\hline & & $0.20-0.40$ & 5.5 & 0.8 & 0.09 & $\operatorname{Tr}^{Q}$ & 1.8 & 1.9 & 0.7 & 12.3 & 2.9 \\
\hline & 6 & $0-0.20$ & 5.5 & 0.9 & 0.08 & 1.1 & 0.9 & 5.0 & 0.5 & 14.6 & 1.8 \\
\hline \multirow{5}{*}{ Donante } & & $0.20-0.40$ & 5.4 & 0.5 & 0.04 & $\mathrm{Tr}^{\mathrm{Q}}$ & 1.5 & 2.3 & 0.6 & 25.5 & 3.2 \\
\hline & 7 & $0-0.20$ & 5.4 & 1.6 & 0.09 & 7.7 & 0.6 & 3.5 & 0.3 & 14.2 & 2.1 \\
\hline & & $0.20-0.40$ & 5.5 & 0.5 & 0.05 & $\operatorname{Tr}^{\mathrm{Q}}$ & 0.8 & 3.4 & 0.5 & 10.3 & 2.6 \\
\hline & 8 & $0-0.20$ & 5.4 & 1.8 & 0.08 & 3.4 & 0.7 & 4.0 & 0.5 & 15.6 & 1.4 \\
\hline & & $0.20-0.40$ & 5.8 & 1.3 & 0.07 & $\mathrm{Tr}^{\mathrm{Q}}$ & 0.8 & 6.0 & 0.7 & 13.1 & 0.6 \\
\hline
\end{tabular}

$\overline{\mathrm{Z}}$ Reacción del suelo. ${ }^{\mathrm{Y}}$ Materia orgánica. ${ }^{\mathrm{X}}$ Nitrógeno. ${ }^{\mathrm{W}}$ Fósforo. ${ }^{\mathrm{V}}$ Potasio. ${ }^{\mathrm{U}}$ Calcio. ${ }^{\top}$ Magnesio. ${ }^{\mathrm{S}}$ Capacidad de intercambio catiónico. ${ }^{\mathrm{R}}$ Acidez intercambiable. ${ }^{\mathrm{Q}}$ Trazas.

Tabla 3. Características morfológicas del suelo en tres puntos del sitio experimental Santa Cruz, Tlahuitoltepec, Oax.

\begin{tabular}{|c|c|c|c|c|c|c|}
\hline \multirow{2}{*}{ Horizonte } & \multirow{2}{*}{$\begin{array}{l}\text { Profundidad } \\
\qquad(\mathrm{cm})\end{array}$} & \multirow{2}{*}{$\begin{array}{l}\text { Color en } \\
\text { húmedo }\end{array}$} & \multirow{2}{*}{ Estructura } & \multicolumn{2}{|c|}{ Consistencia } & \multirow{2}{*}{ Permeabilidad } \\
\hline & & & & Seco & Húmedo & \\
\hline & & \multicolumn{5}{|c|}{ Ladera cóncava parte Norte } \\
\hline Ap & $0-20$ & 10YR4/4 & Bloques subangulares & Suelto & & Moderada \\
\hline Bw & $20-65$ & 7.5YR5/8 & Bloques subangulares & & Friable & Moderada \\
\hline Bt & $65-95$ & 7.5YR5/8 & Bloques angulares & & Firme & Moderada \\
\hline \multirow[t]{2}{*}{$\mathrm{Bt}_{2}$} & $95-126$ & 7.5YR5/8 & Bloques angulares & & Firme & Rápida \\
\hline & & \multicolumn{5}{|c|}{$\begin{array}{l}\text { Ladera convexa parte media } \\
\end{array}$} \\
\hline Ap & $0-15$ & 10YR4/4 & Bloques subangulares & Suelto & & Rápida \\
\hline $\mathrm{Bt}$ & $15-70$ & 10YR4/4 & Bloques subangulares & & Friable & Moderada \\
\hline \multirow[t]{2}{*}{ Bt } & $70-130$ & 7.5YR 5/8 & Bloques angulares & & Firme & Lenta \\
\hline & & \multicolumn{5}{|c|}{ Ladera cóncava parte Sur } \\
\hline Ap & $0-22$ & 10YR5/3 & Bloques subangulares & Suelto & & Moderada \\
\hline Bt & $22-67$ & 10YR6/6 & Bloques subangulares & & Friable & Moderada \\
\hline $\mathrm{Bt}$ & $67-103$ & 10YR6/6 & Bloques angulares & & Firme & Muy lenta \\
\hline
\end{tabular}

El contenido de $\mathrm{MO}$ y el porcentaje de nitrógeno son mayores en el horizonte Ap y menor y variable en los horizontes inferiores. El pH fue de 4.8 en el perfil de la parte Norte y de 4.2 en las partes media y Sur, lo que indica que es un suelo fuertemente ácido. En cuanto a los cationes intercambiables, hubo variación. En la parte Norte, la concentración de $\mathrm{K}$ fue bajo en el horizonte Ap, media en el $\mathrm{Bw}$ y alto en el $\mathrm{Bt}$ y $\mathrm{Bt}_{2}$; en la parte media en los horizontes Ap y Bt fue baja y muy baja en el $\mathrm{Bt}_{2}$; y en parte Sur, fue muy baja en los tres horizontes. El $\mathrm{Ca}$ y $\mathrm{Mg}$ son muy bajos en los tres perfiles. Esto indica que, los cationes se han perdido, lo que ha ocasionado una gran variación de saturación de bases con valores de 53.7 hasta $75.8 \%$, y en la capacidad de intercambio de cationes. En la parte Norte, la CIC fue de 11.3 a $9.9 \mathrm{Cmol}^{(+)} \mathrm{kg}^{-1}$ a través de la profundidad, en la parte media fue de 8.4 en el horizonte Ap y de $7.2 \mathrm{Cmol}(+) \mathrm{kg}^{-1}$ en los horizontes $B t$ y Bt 2 ; y en la parte Sur, en el horizonte $A p$ fue de

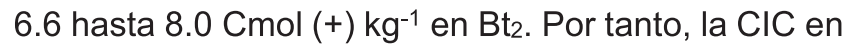
los tres perfiles es baja (Tabla 4). 
Tabla 4. Características físicas y químicas del suelo en la parte Norte, media y Sur del sitio experimental Santa Cruz, Tlahuitoltepec, Oax.

\begin{tabular}{|c|c|c|c|c|c|c|c|c|c|c|c|c|c|c|}
\hline \multirow{3}{*}{ Horizonte } & \multirow{3}{*}{$\begin{array}{l}\text { Profundidad } \\
\qquad(\mathrm{cm})\end{array}$} & \multirow{3}{*}{$\begin{array}{c}\mathrm{Da}^{\mathrm{Z}} \\
\left(\mathrm{g} \mathrm{cm}^{-3}\right)\end{array}$} & \multicolumn{3}{|c|}{ Textura } & \multirow{3}{*}{$\mathrm{pH}^{\mathrm{v}}$} & \multirow{3}{*}{$\begin{array}{l}\mathrm{MO}^{\mathrm{U}} \\
(\%)\end{array}$} & \multirow{3}{*}{$\begin{array}{l}N^{\top} \\
(\%)\end{array}$} & \multirow{3}{*}{$\begin{array}{c}\mathrm{P}^{\mathrm{s}} \\
(\mathrm{mg} \\
\left.\mathrm{kg}^{-1}\right)\end{array}$} & \multicolumn{3}{|c|}{$\begin{array}{c}\text { Cationes } \\
\text { intercambiables }\end{array}$} & \multirow{3}{*}{$\mathrm{ClC}^{\circ}$} & \multirow{3}{*}{$\begin{array}{l}\mathrm{PSB}^{\tilde{N}} \\
(\%)\end{array}$} \\
\hline & & & $A^{Y}$ & $\mathrm{~L}^{\mathrm{X}}$ & $\mathrm{R}^{\mathrm{W}}$ & & & & & $\mathrm{K}^{\mathrm{R}}$ & $\mathrm{Ca}^{\mathrm{Q}}$ & $\overline{\mathrm{Mg}^{\mathrm{P}}}$ & & \\
\hline & & & \multicolumn{3}{|c|}{------- \% ------- } & & & & & ---- & \multicolumn{2}{|c|}{$\mathrm{Cmol}(+) \mathrm{kg}^{-1}$} & & \\
\hline & & \multicolumn{13}{|c|}{ Ladera cóncava parte Norte } \\
\hline Ap & $0-20$ & 1.3 & 32.2 & 36.3 & 31.5 & 4.8 & 5.2 & 0.06 & 0.06 & 0.3 & 0.6 & 0.3 & 11.3 & 53.7 \\
\hline Bw & $20-65$ & 1.4 & 27.6 & 28.3 & 44.0 & 4.7 & 1.5 & 0.02 & 0.06 & 0.4 & 0.1 & 0.1 & 12.4 & 40.7 \\
\hline $\mathrm{Bt}$ & $65-95$ & 1.4 & 38.8 & 23.9 & 37.3 & 4.8 & 0.7 & 0.01 & 0.06 & 0.7 & 0.0 & 0.4 & 9.9 & 59.4 \\
\hline \multirow[t]{2}{*}{$\mathrm{Bt}_{2}$} & $95-126$ & 1.7 & 49.0 & 23.8 & 27.2 & 4.7 & 0.5 & 0.01 & 0.06 & 0.6 & 0.0 & 0.4 & 9.9 & 61.3 \\
\hline & & \multicolumn{13}{|c|}{ Ladera convexa parte media } \\
\hline Ap & $0-15$ & 1.2 & 26.1 & 46.0 & 27.9 & 4.2 & 3.6 & 0.08 & 0.06 & 0.3 & 0.4 & 0.4 & 8.4 & 74.2 \\
\hline $\mathrm{Bt}$ & $15-70$ & 1.3 & 20.1 & 51.5 & 28.4 & 4.3 & 1.3 & 0.04 & 0.06 & 0.2 & 0.0 & 0.2 & 7.2 & 66.4 \\
\hline \multirow[t]{2}{*}{$\mathrm{Bt}_{2}$} & $70-130$ & 1.5 & 18.0 & 41.3 & 40.8 & 4.1 & 0.5 & 0.00 & 0.06 & 0.1 & 0.1 & 0.3 & 7.2 & 72.5 \\
\hline & & \multicolumn{13}{|c|}{ Ladera cóncava parte Sur } \\
\hline Ap & $0-22$ & 1.3 & 11.1 & 59.6 & 29.3 & 4.0 & 4.0 & 0.08 & 0.06 & 0.1 & 0.1 & 0.1 & 6.6 & 75.8 \\
\hline $\mathrm{Bt}$ & $22-67$ & 1.4 & 10.4 & 66.6 & 23.0 & 4.1 & 0.8 & 0.02 & 0.06 & 0.1 & 0.1 & 0.0 & 6.8 & 61.8 \\
\hline $\mathrm{Bt}_{2}$ & $67-103$ & 1.5 & 14.6 & 61.7 & 23.7 & 4.2 & 0.4 & 0.02 & 0.06 & 0.0 & 0.5 & 0.1 & 8.0 & 63.1 \\
\hline
\end{tabular}

Z Densidad aparente. ${ }^{Y}$ Arena. ${ }^{X}$ Limo. ${ }^{W}$ Arcilla. ${ }^{\vee}$ Reacción del suelo. ${ }^{U}$ Materia orgánica. ${ }^{\top}$ Nitrógeno. ${ }^{\mathrm{S}}$ Fósforo. ${ }^{\mathrm{R}}$ Potasio. $\mathrm{Q}$ Calcio. ${ }^{\mathrm{P}}$ Magnesio. ${ }^{\circ}$ Capacidad de intercambio catiónico. ${ }^{\mathrm{N}}$ Porcentaje de saturación de bases.

\subsection{Clasificación}

Las características de los tres perfiles corresponden a un epipedón ócrico de acuerdo con las Claves para la Taxonomía de Suelos (SSS, 2014), dado que cumple con los colores del value en húmedo mayor que 4 en el horizonte Ap (Tablab 3), con un contenido bajo de carbono orgánico. Además con la cantidad de arcilla acumulada en los horizontes Bt y $\mathrm{Bt}_{2}$, el suelo es un Typic Hapludults, del orden Ultisol, que se caracteriza por presentar un horizonte argílico profundo, en el cual se puede obtener dos cosechas al año. De acuerdo a la Base Mundial del Recurso Suelo (WRB, 2007), se clasifica como un Acrisol Háplico (Crómico).

\section{Conclusiones}

En una ladera representativa del agrosistema del municipio de Santa María Tlahuitoltepec, Mixe, Oax., manejada con el sistema MIAF en un periodo de seis años, la zona receptora de sedimentos tuvo modificaciones positivas en las características físicas y químicas respecto a la zona donante, además de la formación de terrazas. Destacando la importancia de aplicar fertilizantes fosfatados y pollinaza, en la mejora de la fertilidad en un suelo fuertemente ácido.

El suelo del agrosistema representativo de la ranchería Santa Cruz, Tlahuitoltepec, Mixe, Oax., se clasifica como Typic Hapludults de acuerdo a la Taxonomía de Suelos y Acrisol Háplico (Crómico) según la clasificación de la FAO. Por lo tanto, el agrosistema se considera como de buena productividad.

Trabajo a futuro. La presente investigación que se derivó de un estudio específico sobre un agroecosistema del estado de Oaxaca, Méx., con el sistema MIAF, permitirá plantear otras formas de abordar la fertilidad de este tipo de suelos bajo este modelo agroecológico de producción.

Agradecimientos. Se agradece a la comunidad étnica Santa María Tlahuitoltepec, por su compresión y apoyo. Al equipo de productividad de agrosistemas del Colegio de Postgraduados y del INIFAP, en pro del avance científico y por último la Fundación Produce Oaxaca, por dar el financiamiento. 


\section{Referencias}

Andrade, B. O. del C. y Rodríguez, P. O. 2002. Evaluación de la eficiencia de barreras vivas como sistemas de conservación de suelos de ladera. Bioagro 14(3): 123133.

Base Referencial Mundial del Recurso Suelo (WRB). 2007. Un marco conceptual para la clasificación, correlación y comunicación internacional. Primera actualización. Informes sobre Recursos Mundiales de Suelos No. 103. FAO, Roma.

Camas, G. R.; Turrent, F. A.; Cortés, F. J. I.; Livera, M. M.; González, E. A.; Villar, S. B.; López, M. J.; Espinoza, P. N. y Cadena, I. P. 2012. Erosión del suelo, escurrimiento y pérdida de nitrógeno y fósforo en laderas bajo diferentes sistemas de manejo en Chiapas, México. Revista Mexicana de Ciencias Agrícolas 3(2): 231-243.

Camas, G. R. 2011. Erosión hídrica, productividad, captura de carbono y uso de la radiación solar y agua en tres sistemas de manejo, para la agricultura de ladera en el trópico subhúmedo de México. Tesis Doctoral. Colegio de Postgraduados, Montecillo, Texcoco, Edo. México.

Cortés, F. J. I.; Torres, Z. J. P; Turrent, F. A.; Hernández, R. E.; Ramos, S. A. y Jiménez, S. L. 2012. Manual actualizado para el establecimiento y manejo del sistema Milpa Intercalada con Árboles Frutales (MIAF) en Laderas. Colegio de Postgraduados, México.

Cortés, J. I.; Turrent, A.; Díaz, P.; Jiménez, S.; Hernández, E. and Mendoza, R. 2005. Hillside agriculture and food security in Mexico: advances in the sustainable hillside Management project. pp. 569-588. In: R. Lal, N. Uphoff, B. A. Stewart, and D. O. Hansen. Climate change and global food security. Taylor and Francis. New York, NY, USA.

Cortés, J. I.; Mendoza, R.; Hernández, E.; Aceves E.; Turrent, A. y Estrella, N. 2004. Manual para Técnicos. El sistema Milpa Intercalada en Árboles Frutales (MIAF) en terrenos planos. Colegio de Postgraduados, Puebla, México. $70 \mathrm{p}$.

Cuanalo, de la C. H. 1981. Manual para la descripción de perfiles de suelo en el campo. 2a ed. Colegio de Postgraduados. Chapingo, Edo. México. 40 p.

Dalton, P. A.; Smith, R. J. and Troung, P. N. V. 1996. Vetiver grass hedges for erosion control on a cropped flood plain: hedge hydraulics. Agricultural water management 31: 91-104.

Etchevers, B. J. D. 1992. Manual de métodos para el análisis de suelos, plantas, aguas y fertilizantes. Análisis rutinarios en estudios y programas de fertilidad.
Laboratorio de Fertilidad, Colegio de Postgraduados. Montecillo, Edo. México.

Fassbender H. W. 1986. Química de suelos, con un énfasis de suelos de América Latina. Instituto interamericano de cooperación para la agricultura. San José, Costa Rica.

García, E. 2004. Modificaciones al sistema de clasificación climática de Köppen (para adaptarlo a las condiciones de la República Mexicana). $5^{a}$ ed. México D. F. 98 p.

Instituto Nacional de Estadística y Geográfica (INEGI). 2010. Prontuario de información geográfica municipal de los Estados Unidos Mexicanos: Santa María Tlahuitoltepec, Oaxaca.

Lott, J.; Khan, A.; Black, C. and Ong, C. 2003. Water use in a Grevillea robusta-maize overstory agroforestry system in semi-arid Kenya Forest Ecology and Management 180: 45-59.

Martínez, G. A. y Martínez, D. M. A. 1996. Diseños de experimentos con fertilizantes. Publicación especial 5. Sociedad Mexicana de la Ciencia del Suelo A. C y Colegio de Postgraduados. Chapingo, México. 155 págs.

Medina, O. L. E.; Bravo, E. M.; Prat, Ch.; Martínez, M. M.; Ojeda, T. E. y Serrato, B. B. E. 2008. Pérdida de suelo, agua $y$ nutrientes en un Acrisol bajo diferentes sistemas agrícolas en Michoacán, México. Agricultura Técnica en México 34(2): 201-211.

Ojo, A. O.; Adetunji, M. T.; Okeleye, K. A. and Adejuyigbe, C. O. 2015. Soil fertility, phosphorus fractions, and maize yield as affected by poultry manure and single superphosphate. Hindawi Publishing Corporation. International Scholarly Research Notices. Volume 2015, Article ID 616213, 8 pages.

Ortiz, S. C. A.; Suástegui, M. E. y Alonso, A. D. 2011. Normales climatológicas 1951-2010. Secretaria del Medio Ambiente y Recursos Naturales. Comisión Nacional del Agua. Colegio de Postgraduados, Montecillo, Méx.

Pool, N. L.; Trinidad, S. A.; Etchevers, B. J. D.; Pérez, M. J. y Martínez, G. A. 2000. Mejoradores de la fertilidad del suelo en la agricultura de ladera de los altos de Chiapas, México. Agrociencia 34(3): 251-259.

Ramos, S. A., Cortés, F. J. I., Turrent, F. A., Jiménez, S. L., Torres, Z. J. P., y Martínez, M. O. 2007. Proyecto Colegio de Postgraduados-Fundación Produce Oaxaca: Investigación agronómica y transferencia de tecnología en la fase de escalamiento del PMSL en el estado de Oaxaca. $1^{\text {a }}$ Etapa. 
Ryugo, K. 1993. Fruticultura. Ciencia y arte. Traducción al español de J. Rodríguez A. AGT Editor, S. A. México, D. F. p. 227.

Salinas, S. R. 2015. Caracterización topográfica, física y de fertilidad de un suelo de ladera manejado con el sistema milpa intercalada con árboles frutales durante cinco años. Tesis de Maestría en Ciencias. Colegio de Postgraduados, Montecillo, Texcoco. Edo. de México.

Santiago, M. E. 2006. Estado nutrimental y rendimiento del duraznero (Prunus persica (L) Batsch) en el sistema milpa intercalada con árboles frutales en laderas pronunciadas. Tesis de Maestría en Ciencias. Colegio de Postgraduados, Montecillo, Texcoco. Edo. de México.

Sanyal, S. K.; Dwivedi, B. S.; Singh, V. K.; Majumdar, K.; Datta, S. C.; Pattanayak, S. K. and Annapurna, K. 2015. Phosphorus in relation to dominant cropping sequences in India: chemistry, fertility relations and management options. Current Science 108(7): 12621270.

Secretaría del Medio Ambiente y Recursos Naturales (SEMARNAT). 2000. Norma Oficial Mexicana NOM021-RECNAT-200, que establece las especificaciones de fertilidad, salinidad y clasificación de suelos, estudio, muestreo y análisis. Diario Oficial. México.

Shibu, J. 2009. Agroforestry for ecosystem services and environmental benefits: an overview. Agroforestry systems. 76: 1-10.

Smith, J.; Pearce, B. D. and Wolfe, M. S. 2012. Reconciling productivity with protection of the environment: Is temperate agroforestry the answer? Renewable Agriculture and Food Systems 28(1): 80-92.

Soil Survey Staff (SSS). 2014. Keys to Soil Taxonomy. 12th ed. USDA-Natural Resources Conservation Service, Washington, DC.

Steppler, H. A. and Nair, P. K. R. 1987. Agroforestry a decade of development. International Council for Research in Agroforestry (ICRAF). Nairobi, Kenya.
Tornquist, C. G.; Honsb, F. M.; Feagleyb.S, E. and Haggar, J. 1999. Agroforestry system effects on soil characteristics of the Sarapiquí region of Costa Rica. Agriculture, Ecosystems and Environment 73: 9-28.

Torres, Z. J. P. 2006. Rendimiento de fruto y calidad de fruto de durazno en función del número de ramas de estructura bajo el sistema milpa intercalada con árboles frutales. Tesis de Maestría en Ciencias. Colegio de Postgraduados, Montecillo, Texcoco, Edo. México.

Turrent F. A.; Francisco N. N.; Uribe G. F. y Camacho, C. R. 1998. La terraza de muro vivo, tecnología para la explotación prosostenible de laderas roturadas del Trópico subhúmedo de México. Agricultura Técnica en México. 24 (1): 67-81.

Turrent A., Uribe S., Francisco N. y Camacho R. 1995. La terraza de muro vivo para laderas de trópico subhúmedo de México. I Análisis del desarrollo de las terrazas durante 6 años. Terra 13(3): 276-298.

United States Department of Agriculture (USDA). 2011. Newhall 1.5.1. Natural Resources Conservation Service, Penn State University Center for Environmental Informatics.

Van Reeuwijk, L. P. 1999. Procedimientos para el Análisis de Suelos, versión 1995. Traducido de: Ma. del Carmen Gutiérrez Castorena, Carlos Arturo Tavares Espinoza y Carlos Alberto Ortiz Solorio. Primera Edición, 1999. Especialidad de Edafología, Colegio de Postgraduados, Montecillo, México. 145 págs.

Vergara, S, M. A.; Etchevers, B, J. D. y Padilla, C. J. 2005. La fertilidad de los suelos de ladera de la sierra Norte de Oaxaca, México. Agrociencia 39: 259-266.

Volke, H. V.; Turrent, F. A. y Castillo, M. A. 2005. Diseños de tratamientos y estimación de funciones de respuesta en la investigación agrícola. Colegio de Postgraduados, Montecillo, Estado de México, México. 68 págs. 